\title{
Caractérisation expérimentale de la rupture en mode localisé d'un grès
}

\section{A. HAIED \\ D. KONDO J.-P. HENRY}

Laboratoire de Mécanique de Lille, URA CNRS 1441.

Université Lille 1,

59655 Villeneuve-d'Ascq

Cedex
Cette étude expérimentale porte sur la localisation des déformations dans le grès de Fontainebleau. Après avoir rappelé le comportement mécanique du grès, nous présentons la procédure expérimentale de détection de la localisation. Cette procédure, basée sur des mesures multiples de déformations, permet par comparaisons des mesures de préciser les conditions de perte

d'homogénéité des déformations dans les échantillons testés. Une première application permet de montrer que l'efficacité de la méthode est limitée dans le cas des essais triaxiaux de révolution (éprouvettes cylindriques), la détection du seuil de non homogénéité des déformations n'étant pas systématique, L'utilisation d'échantillons prismatiques avec ou sans imperfection, ce qui constitue l'originalité de cette étude, améliore de façon substantielle l'efficacité de la procédure. Les caractéristiques des bandes de localisation (seuil, orientation, épaisseur) sont alors fournies et discutées pour l'ensemble de ces essais triaxiaux sur éprouvettes prismatiques.

\section{Experimental characterization of localized failure mode in a sandstone}

The paper concerns an experimental study of strain localization in Fontainebleau sandstone. After recalling the mechanical behaviour of the sandstone, we describe the experimental procedure for detecting strain localization. This is based on multiple measurements of strains and displacements, which by comparisons give indication on strain inhomogeneity. First tests are performed on cylindrical samples and it is shown that the procedure is not systematically efficient on this kind of experiments. The original feature of the present study is the use of prismatic samples which provides meaningful data concerning strain localization in brittle rocks. In order to control localization initiation, a last serie of tests is performed on prismatic samples containing a little hole. Shear bands characteristics (onset, orientation, thickness) are given for all these triaxial tests on prismatic sample. 


\section{Introduction}

Les problèmes d'instabilité de structures en milieu rocheux ont depuis longtemps été au centre des préoccupations des ingénieurs et exploitants en charge de ces structures. L'analyse des phénomènes de rupture dans les roches indique souvent une localisation de la déformation, inexplicable par les approches conventionnelles. L'application récente de la théorie de la bifurcation (Rudnicki et Rice, 1975) aux problèmes de localisation a ouvert la vole à une approche plus rationnelle de ces phénomènes de rupture. Le critère de localisation de Rice permet de prédire dans le cas des sollicitations quasi statiques les conditions de localisation de déformations (seuil, orientations des surfaces de localisation). La théorie de la bifurcation a également suscité d'importants travaux expérimentaux en géomécanique. Les premières études dans ce domaine concernaient surtout les sols (cf. p. ex. Vardoulakis et Graf, 1982; Desrues, 1984). En mécanique des roches, beaucoup de travaux ont porté auparavant sur le rôle de la microfissuration dans l'apparition de la rupture. La plupart d'entre eux indiquent notamment qu'à l'approche de la rupture les microfissures s'organisent en réseaux, formant ainsi des zones de densité de microfissuration élevée. Les études spécifiques de localisation dans les roches ou les bétons sont plus récentes et peu nombreuses (cf. p. ex. Santarelli, 1990; Ord et al., 1991; Haied et al., 1993; Bascoul et al., 1993). L'objectif de la présente étude est de caractériser (quantitativement et qualitativement) la localisation des déformations dans le grès de Fontainebleau. Nous avons privilégié la simplicité des moyens expérimentaux en utilisant la technique des mesures multiples de déformations, en particulier sur des éprouvettes prismatiques.

\section{2}

\section{Résumé du comportement mécanique du grès en compression triaxiale de révolution}

\section{1}

\section{Matériau utilisé, conditions d'essai et protocole expérimental}

En raison de sa simplicité chimique et cristallographique et de son intérêt pour l'industrie pétrolière, le grès de Fontainebleau a été retenu pour cette étude. De plus dans un tel matériau, constitué essentiellement de grains de quartz (98\%), les microfissures sont relativement faciles à observer, ce qui constitue un avantage pour l'étude microscopique (ultérieure) des phénomènes de localisation. Ce grès a une porosité comprise entre 9 et $11 \%$ et un poids spécifique de 23,7 $( \pm 0,2) \mathrm{kN} / \mathrm{m}^{3}$.

Les éprouvettes utilisées pour la réalisation des essais de caractérisation mécanique sont des cylindres droits à base circulaire. Ils sont carottés dans un même bloc de grès de Fontainebleau sain. Les carottes ont un diamètre de $37,5 \mathrm{~mm}$, la hauteur des éprouvettes est limitée à $75 \mathrm{~mm}$. Une rectifieuse est utilisée pour le trai- tement des surfaces de chargement. Les imperfections du polissage des surfaces de chargement sont de moins de $5 \mu \mathrm{m}$; la tolérance sur le parallélisme des deux surfaces est inférieure à $10 \mu \mathrm{m}$, ce qui respecte la valeur exigée et recommandée par la Société internationale de mécanique des roches (SIMR). Six jauges de déformations sont collées à mi-hauteur des éprouvettes testées. Deux d'entre elles sont destinées à mesurer les déformations longitudinales, elles sont de longueur $5 \mathrm{~mm}$ et sont collées le long de deux génératrices diamétralement opposées. Les quatre autres, de longueur $2 \mathrm{~mm}$, disposẻes perpendiculairement aux génératrices permettent la mesure des déformations latérales.

Pour la réalisation des essais triaxiaux, la pression de confinement est appliquée à l'aide d'une pompe de type «Gilson » ( $1 \mathrm{MPa} / \mathrm{mn})$. Le chargement axial est réalisé par asservissement en déplacement axial du vérin, avec une vitesse de déformation fixée à $5 \cdot 10^{-6} / \mathrm{s}$. Les essais ont été réalisés à la température ambiante (18$22^{\circ} \mathrm{C}$ ).

\section{2}

\section{Comportement mécanique de base du grès}

Sous compression hydrostatique, les réponses longitudinale et transversale du matériau sont identiques. Ceci indique que le grès étudié est un matériau initialement isotrope. Les résultats donnent une faible pression de fermeture $(P, \approx 2 M P a)$ et une porosité de fissure d'environ $1,6.10^{-4}$.

\section{Q19.9.}

\section{Essais de compression triaxiale monotone}

Les essais triaxiaux de révolution ont été réalisés pour des pressions de confinement allant jusqu'à $30 \mathrm{MPa}$. La limitation à $30 \mathrm{MPa}$ est tributaire des capacités de la presse utilisée. Les réponses du matériau en compression triaxiale de révolution correspondent schématiquement à ce que l'on obtient classiquement pour le comportement fragile des roches (Bieniawski, 1967): (i) phase de fermeture des pores et microfissures préexistantes; (ii) phase de déformations élastiques linéaires; (iii) phase d'amorçage et de propagation stable de la microfissuration dont le début est marqué par le seuil de dilatance. La linéarité des courbes contraintes-déformations axiales au cours de cette phase indique la tendance des microfissures à se développer perpendiculairement à la direction de la contrainte principale mineure; (iv) phase de propagation instable de microfissures qui débute à partir du niveau de contrainte où le taux de déformation volumique change de signe et un peu avant le pic de contraintes. C'est au cours de cette phase que se produisent probablement les interactions entre réseaux de microfissures à l'origine de la localisation des déformations dans les roches fragiles.

\subsection{4.}

\section{Observations sur les surfaces de rupture}

A partir des observations des éprouvettes rompues, il a été possible de distinguer plusieurs types de ruptures. Dans l'essaí uniaxial, la rupture se développe le plus souvent selon des plans parallèles à l'axe de chargement. En présence de pression de confinement 
modérée $(20 \mathrm{MPa})$ ou relativement élevée ( $30 \mathrm{MPa}$, la rupture se développe d'une manière classique le long d'un plan unique faisant un angle d'environ $31-32^{\circ}$ avec la direction de la contrainte principale majeure.

\section{3}

\section{Détection expérimentale de la localisation des déformations}

\section{1}

\section{Principe de la méthode de détection}

La technique utilisée pour déterminer le seuil de localisation est inspirée des travaux de Hadley (1975). En effet, dans l'essai triaxial sur un granite et un gabbro, cet auteur a tracé l'ellipse moyenne des déformations circonférentielles. Aux valeurs élevées des contraintes, l'auteur observe que, contrairement aux cas des faibles nivaux de contraintes, les points de mesures (obtenus dans plusieurs directions radiales) s'écartent remarquablement de l'ellipse de moyenne caractérisant un champ de déformations homogène. C'est cette non-homogénéité des déformations qui sera considérée comme critère expérimental d'apparition de la localisation. Si la mise en œuvre pratique de la méthodologie consiste simplement à réaliser des mesures multiples de déformations (par capteurs ou par jauges d'extensométrie), elle requiert néanmoins un soin et une précision remarquable dans les mesures.

Santarrelli (1990) a utilisé avec succès cette technique dans l'essai triaxial de révolution en comparant les déformations radiales ( $\varepsilon_{i 2}$ et $\varepsilon_{p a}$ ) mesurées dans deux directions orthogonales entre elles. En pratique, la comparaison des déformations est réalisée en utilisant la courbe de variation des déformations $\varepsilon_{r 3}$ en fonction de $\varepsilon_{r 2}$. Le seuil de localisation est alors supposé correspondre à la perte de linéarité de cette courbe. Soulignons que d'autres auteurs tels que Ord et al. (1991) ont également utilisé des procédures expérimentales basées sur des mesures multiples de déformations. Dans nos essais axisymétriques, les mesures ont été effectuées dans la section droite située à mi-hauteur de l'éprouvette. Ce choix s'appuie sur les observations des éprouvettes rompues qui indiquaient un endommagement important au centre des échantillons.

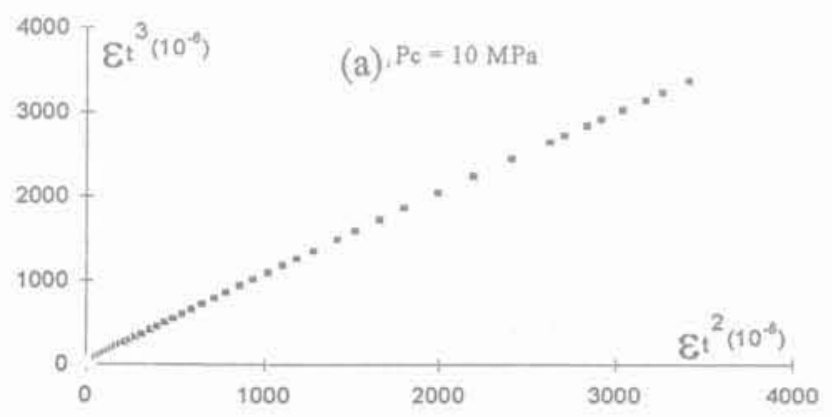

\section{2}

\section{Localisation dans les essais triaxiaux de révolution}

Les quatre jauges de déformations transversales sont donc collées à mi-hauteur de l'éprouvette, perpendiculairement à quatre génératrices diamétralement opposées deux à deux. Chaque paire de jauges assure la mesure de la déformation le long d'une ligne perpendiculaire à la ligne diamétrale qui joint les points milieux des jauges. La moyenne des deux mesures dans chacune des deux directions est respectivement $\varepsilon_{1}^{2}$ et $\varepsilon_{1}^{3}$. Ces deux directions sont a priori équivalentes et, pour une présentation cohérente des résultats, on notera conventionnellement $\varepsilon_{1}^{3}$ la direction où la déformation maximale (enregistrée à la rupture macroscopique) est la plus élevée.

Deux exemples de variation de $\varepsilon_{t}^{3}$ en fonction de $\varepsilon_{i}^{2}$ sont présentés pour différentes pressions de confinement sur la figure 1. Les courbes obtenues présentent une partie linéaire de pente 1. Ce résultat, escompté, indique deux points essentiels dans cette étude. Il s'agit d'une part de l'homogénéité des essais et, d'autre part, de l'isotropie transverse du matériau dans les premières phases de déformation. A partir de ces exemples de courbes, on distingue deux types de réponse. Le premier (Fig. 1b) est celui de l'essai triaxial mené à $30 \mathrm{MPa}$ de confinement (c'est aussi le cas de l'essai uniaxial) où on observe, au-delà d'un seuil, une déviation par rapport à la linéarité de la courbe $\left(\varepsilon^{3}, \varepsilon^{2}\right)$. Le second (Fig. 1a) est celui relatif à l'essai triaxial mené à $10 \mathrm{MPa}$ (c'est aussi le cas à $20 \mathrm{MPa}$ de confinement) pour lequel la linéarité se poursuit jusqu'au pic. Si on s'intéresse au premier type de réponse, le point précis où la perte de linéarité de la courbe $\varepsilon_{t}^{3}, \varepsilon_{1}^{2}$ a lieu (point noté u $A$ ) sera désigné upseudo-seuil de localisation », puisque son observation n'est pas systématique dans tous les essais sur éprouvettes cylindriques. Reporté sur les courbes contraintes-déformations, ce point A se situerait autour de $88 \%$ de la résistance à la rupture dans le cas de l'essai de compression uniaxiale et à $90 \%$ dans l'essai triaxial à $30 \mathrm{MPa}$ de confinement.

Parce que le second type de réponse donne un écart à la linéarité insuffisamment marqué pour justifier de la non-homogénéité des déformations, on doit donc conclure que la méthodologie expérimentale n'est pas systématiquement performante dans le cas des essais axisymétriques. Cela est sans doute lié au choix hasardeux (vis-à-vis de la future bande de localisation) des positions oủ les déformations sont mesurées.

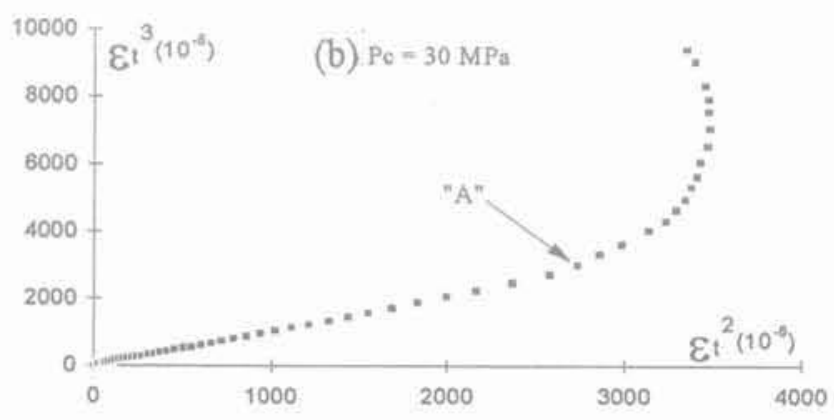

FG.1 Détection du seuil de localisation dans les essais triaxiaux de révolution. Courbes $\varepsilon^{2}, \varepsilon^{3}$. Le point $\mathrm{A}$ sur la figure $1 \mathrm{~b}$ indique le seuil de perte d'homogénéité des déformations.

Localisation in triaxial axisymetric tests. Curves $\varepsilon^{2}, \varepsilon^{3}$, Point $A$ on figure $1 \mathrm{~b}$ indicates the onset of strain inhomogeneity. 


\section{Quelques remarques et conclusions}

Nous venons de souligner que la difficulté à situer le seuil de localisation dans l'essai triaxial de révolution provient principalement du choix hasardeux des deux directions de mesures de $\varepsilon_{,}^{2}$ et $\varepsilon_{1}^{3}$ par rapport à la future bande (qui se présente généralement selon un plan unique). La détection du seuil de localisation par mesures multiples de déformations dans les essais triaxiaux de révolution est donc conditionnée par l'orientation du plan de rupture. Une première idée d'amélioration substantielle a consisté à mener la suite des investigations expérimentales sur des éprouvettes prismatiques. De plus, cette forme d'éprouvette permet également l'utilisation d'un collier de mesure de déformations latérales globales, spécifiquement mis au point pour cette partie de l'étude et destiné à améliorer la précision des mesures.

\section{3}

\section{Essais sur éprouvettes prismatiques}

\subsection{1}

\section{Définitions géométriques des échantillons prismatiques et instrumentation}

Les essais présentés dans ce paragraphe sont menés sur éprouvettes prismatiques. La forme prismatique, choisie, offre un certain nombre d'avantages. Le premier est la connaissance préalable des plans dans lesquels est susceptible d'apparaitre la bande de localisation. Ces plans sont bien entendu définis par la direction de chargement et l'une ou l'autre des directions latérales (mieux définies que dans le cas de la géométrie axisymétrique). L'autre intérêt, concerne la levée de l'ambiguité sur la mesure des déformations dans chacune des trois directions de l'espace. Tous ces avantages constituent l'originalité de cette étude.
Les échantillons, prélevés dans un même bloc, sont découpés dans une matrice de marbrier les dotant de dimensions géométriques quasi identiques. La rectification des surfaces (inférieure à $5 \mu \mathrm{m}$ ), la réalisation du parallélisme et de l'orthogonalité des faces sont similaires à celles indiquées pour les éprouvettes cylindriques. Les dimensions adoptées pour la confection des éprouvettes prismatiques tiennent compte de deux facteurs:

(i) la limitation de l'épaisseur et de la largeur des éprouvettes est dictée par les dimensions du support solidaire au socle de la cellule triaxiale que nous avons utilisée, le support à l'intérieur de cette cellule étant initialement destiné à servir d'embase inférieure et à la mise en place d'échantillons cylindriques de diamètre $65 \mathrm{~mm}$;

(ii) l'usage d'éprouvettes de faible épaisseur expose à des instabilités de type flambement.

Pour ces raisons, nous avons choisi une hauteur d'environ $90 \mathrm{~mm}$ pour une largeur d'environ $60 \mathrm{~mm}$ et une épaisseur de $12 \mathrm{~mm}$ (voir figure 2). Sur chacune des éprouvettes, sont placées huit jauges (Fig. 2): trois jauges dans la direction 1 (longitudinale), deux dans la direction 2 (transversale) et trois dans la direction 3 (latérale). Les deux jauges situées au centre de l'éprouvette sont destinées à mesurer la déformation moyenne de l'échantillon dans les directions longitudinale et latérale. Elles doivent également permettre d'évaluer les déformations locales au centre de l'éprouvette où est supposé apparaitre la bande de localisation. Les six autres jauges qui peuvent être divisées en deux groupes de trois sont destinées aux mesures des déformations dans chacun des deux blocs que la bande est sensée séparer. La comparaison des diverses déformations mesurées dans la même direction en des points différents de la surface de l'éprouvette a, en pratique, permis de s'assurer de la cohérence des mesures au cours du test.

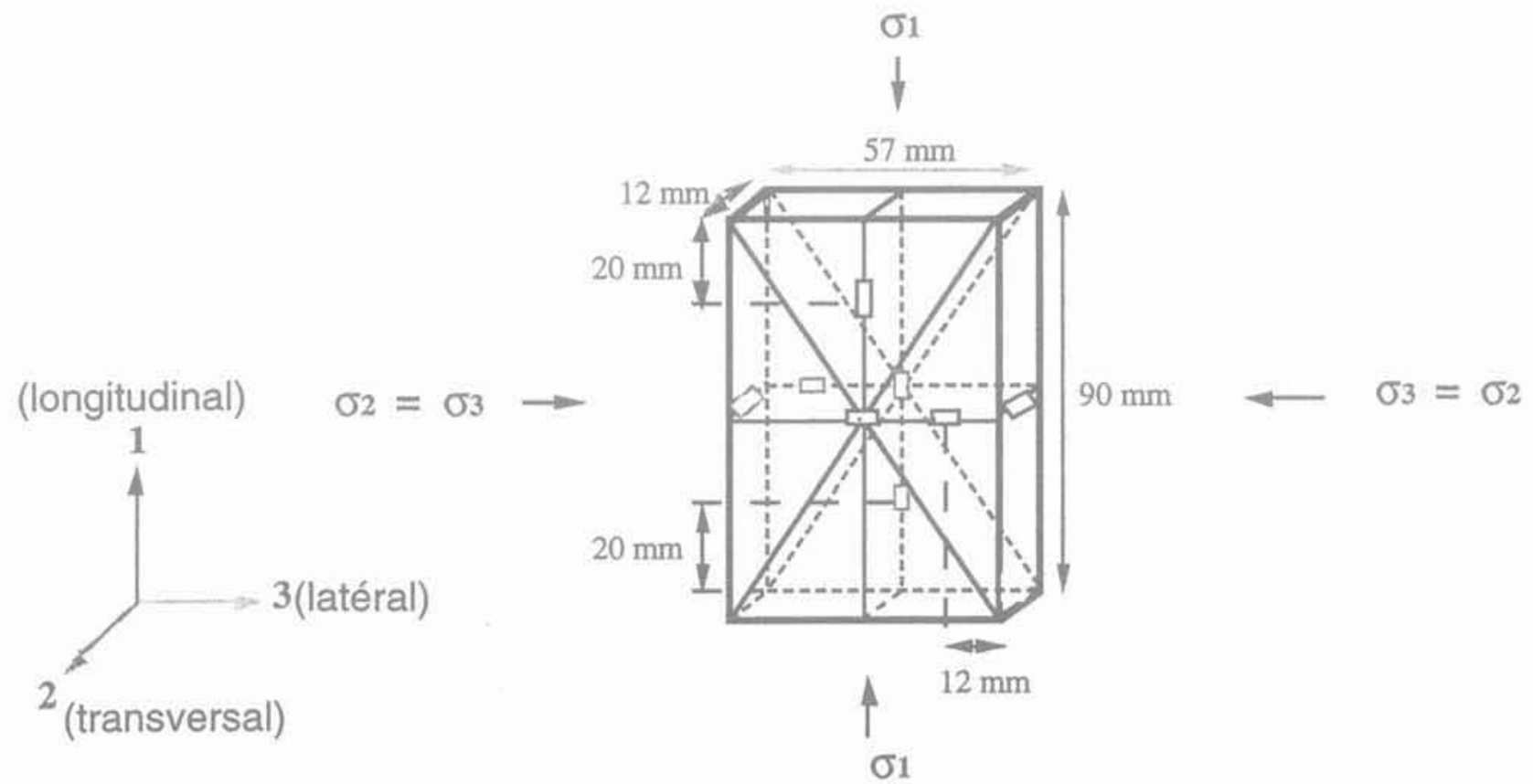

FG. 2 Définition géométrique des éprouvettes prismatiques et disposition des jauges. Geometrical definition of prismatic samples and strain gages disposition. 


\section{3 .2}

\section{Réponses mécaniques et observations sur les surfaces de rupture}

L'asservissement est réalisé en déplacement axial contrôlé, la vitesse de déplacement étant la même que celle utilisée dans les essais axisymétriques $\left(5 \cdot 10^{-6} \cdot \mathrm{s}^{-1}\right)$. Les essais triaxiaux sont réalisés aux pressions de confinement de $0,10,20,30$ et $40 \mathrm{MPa}$. Un exemple de variations des déformations axiales $\left(\varepsilon_{j}\right)$, transversales $(\varepsilon)$, latérales $\left(\varepsilon_{3}\right)$ et volumiques $\left(\varepsilon_{v}\right)$ en fonction du déviateur de contraintes est présenté sur la figure 3 (essai à $20 \mathrm{MPa}$ de confinement). Les résultats des autres essais peuvent être trouvés dans Haied (1995). Ces courbes ont une allure classique, similaire à celle des essais axisymétriques. On notera cependant ici une différence entre les valeurs des déformations transversales $\varepsilon$, et celles (plus élevées) des déformations latérales $\varepsilon_{3}$. On peut en partie attribuer ceci à la géométrie de l'éprouvette qui n'est pas tout à fait tridimensionnelle (l'épaisseur étant relativement faible) ou à la nature très locale des mesures de $\varepsilon_{2}$.

L'examen macroscopique des lignes de rupture des éprouvettes prismatiques rompues montrent que le passage du mode de rupture axiale à la rupture en bande inclinée est obtenue dès la pression de confinement de $10 \mathrm{MPa}$. On peut retenir que dans l'essai triaxial, la rupture a lieu en bande de cisaillement inclinée d'environ 29 à $32^{\circ}$ par rapport à l'axe de chargement, cette orientation augmentant progressivement avec la pression de confinement. On note une diminution appréciable des largeurs de bande en fonction de la pression de confinement; ces largeurs de bande varient d'un peu plus de $2 \mathrm{~mm}$ dans l'essai de compression triaxiale mené à $10 \mathrm{MPa}$ de confinement à moins de $1 \mathrm{~mm}$ dans l'essai à $40 \mathrm{MPa}$ de confinement.

\section{3 .3}

\section{Déformations non homogènes et seuil de localisation}

Des exemples de courbes $\varepsilon_{2}, \varepsilon_{3}$ sont donnés sur la figure 4. Elles montrent toutes une déviation par rapport à la linéarité. Contrairement aux pseudo-seuils obtenus dans les essais triaxiaux de révolution, le point de non-linéarité noté $\mathrm{A}$, que l'on détermine ici sans ambiguitté, peut être considéré comme le seuil de localisation. La pente de la partie linéaire des courbes est supérieure à 1: elle se maintient à environ 1,7 dans l'essai uniaxial et dans les essais triaxiaux menés à 10 et $20 \mathrm{MPa}$ de confinernent et diminue à 1,3 puis 1,2 dans respectivement les essais réalisés à 30 et $40 \mathrm{MPa}$ de confinement. La déviation par rapport à la linéarité provient d'une augmentation soudaine des déformations $\varepsilon_{\text {f }}$ (fissuration dans la bande probablement axiale et dans le plan de l'éprouvette) qui se poursuit jusqu'à la rupture. Cette augmentation est supposée marquer le seuil des déformations non homogenes et l'amorçage d'une zone de déformation localisée au sein du matériau. Soulignons également, comme Santarelli (1990), que la plus grande partie de la dilatance se développe après le seuil de localisation.

Dans l'essai uniaxial, le seuil de localisation A se situe à $96 \mathrm{MPa}$, soit à $88 \%$ de la résistance à la rupture. Cette valeur est en parfaite concordance avec le pseudo-seuil de localisation déterminé dans l'essai axisymétrique, Dans les essais triaxiaux le seuil est enregistré entre 82 et $76 \%$ du pic (voir figure 3 pour l'essai à $20 \mathrm{MPa}$ de confinement). Le point B (seuil de propagation instable de microfissures défini par Bieniawski (1967)), où la pente de la courbe de déformations volumiques change de signe, précède systématiquement le seuil de localisation. Si on s'intéresse aux valeurs des

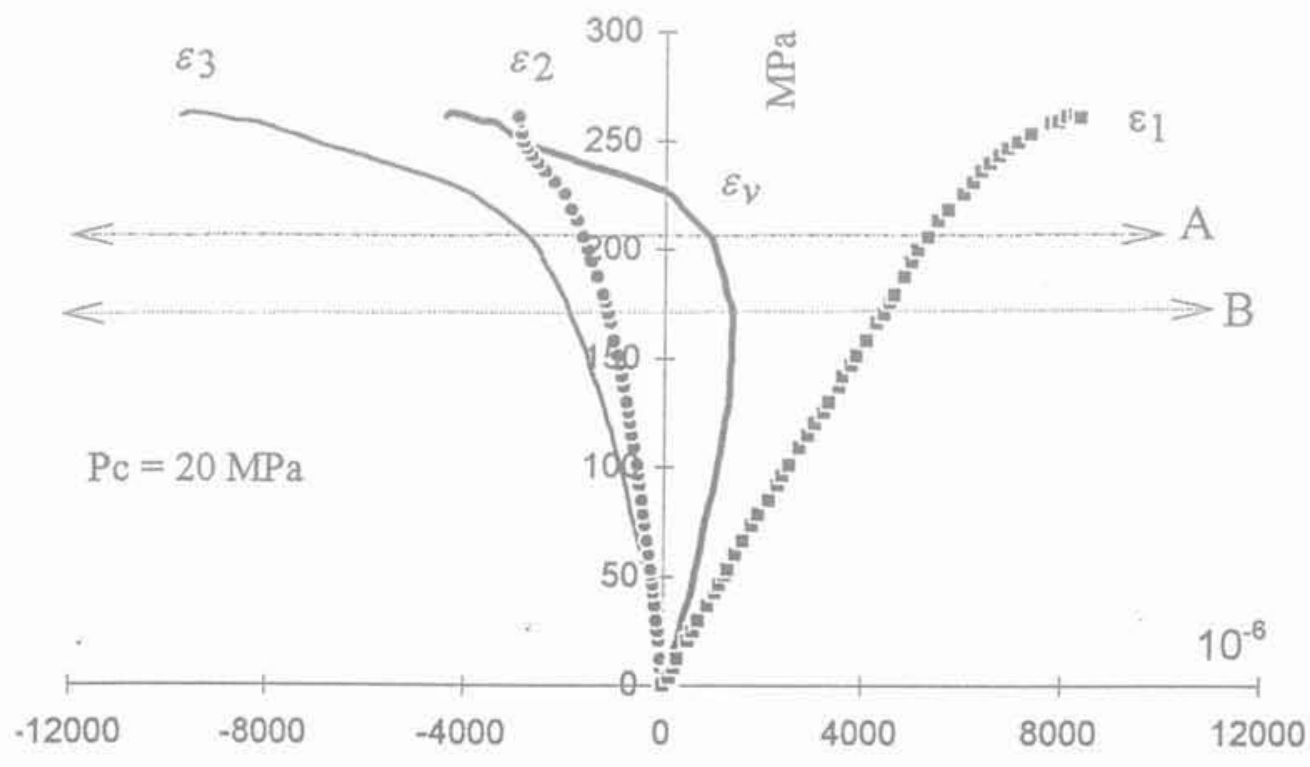

FIG.3 Exemple de réponse en compression triaxiale sur éprouvette prismatique. Example of stress-strain curve for triaxial test on prismatic samples. 

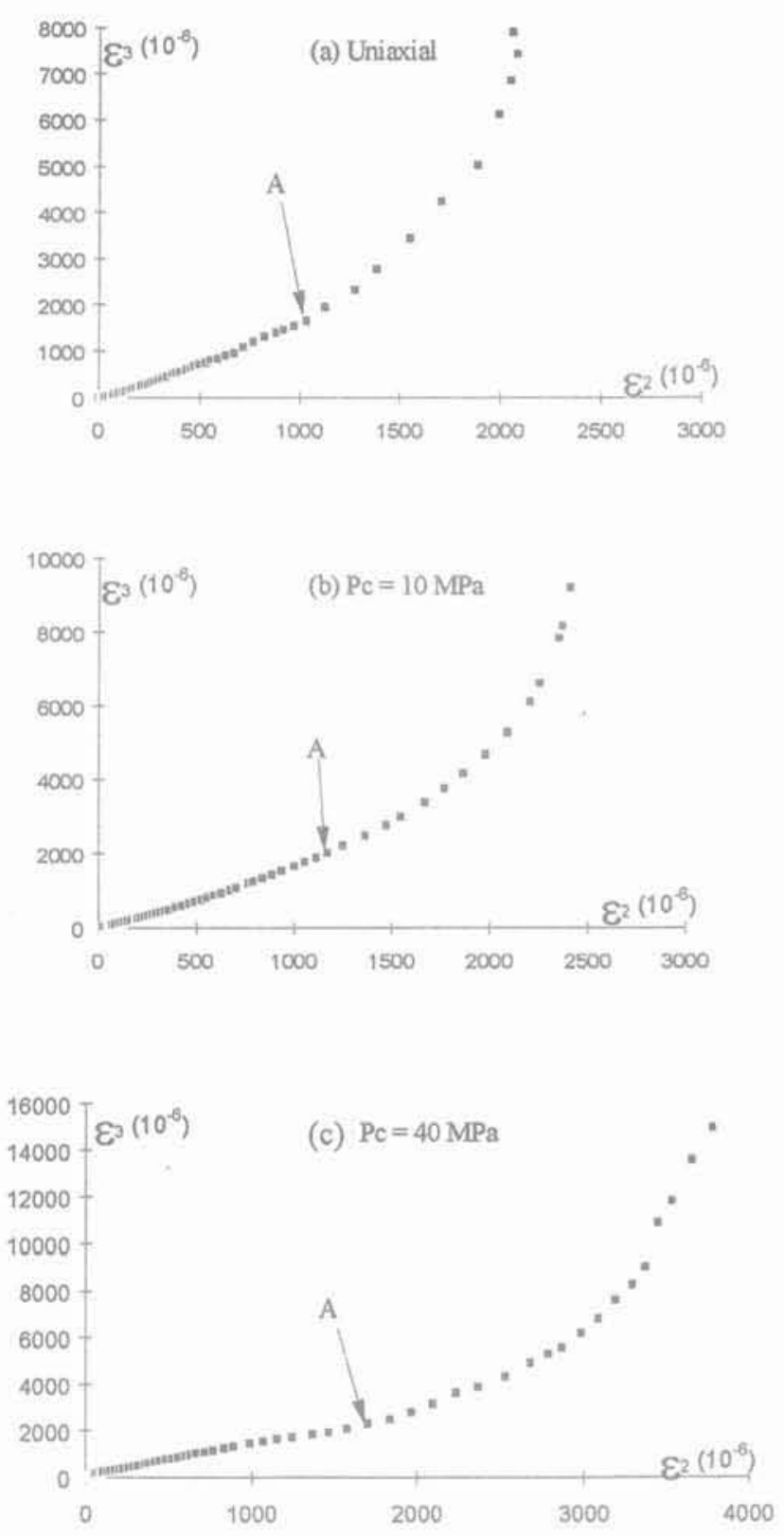

FIG. 4 Courbes $\varepsilon_{2}, \varepsilon_{3}$ dans les essais triaxiaux sur éprouvetté prismatique. «A $\mathrm{A}$ indique le seuil de perte d'homogénéité des déformations dans les essais.

Curves $\varepsilon_{2}, \varepsilon_{2}$ in prismatic samples. Point A indicates the onset of strain inhomogeneity.

déformations enregistrées aux seuils de localisation dans les trois directions, on constate que ces seuils apparaissent pour des niveaux de déformations souvent comparables; nous reviendrons sur ce point à propos des essais sur éprouvettes percées que nous abordons maintenant.

\section{4}

\section{Contrôle et suivi des bandes de localisation}

La nécessité de contrôler l'amorçage des bandes de cisaillement et également de suivre leur évolution durant la phase post localisation est un des objectifs que nous nous sommes fixés dans cette étude. De plus, lorsqu'on décharge les éprouvettes prismatiques précédentes après les avoir sollicitées légèrement au-dessus du seuil de localisation, aucune trace visible (à l'œeil nu) de rupture ou d'endommagement local n'est repérable sur la surface de l'éprouvette. Ceci est probablement dû au fait que la zone de localisation se développe en premier lieu en cœur plutôt qu'en peau des éprouvettes (Haied, 1995) ou à la taille très faible des microfissures. La démarche retenue pour contrôler la zone d'amorçage des bandes a consisté à utiliser des éprouvettes prismatiques percées au centre d'un trou cylindrique de faible diamètre ( $3 \mathrm{~mm}$ ), d'axe perpendiculaire à l'axe de chargement et traversant l'éprouvette sur toute son épaisseur (Fig. 5). Cette idée n'est pas complètement nouvelle; en effet, on trouve trace d'essais du même type sur des métaux ou sur des roches dans l'ouvrage de Nadai (1950). Vardoulakis et Graff (1982) ont également utilisé soit une encoche (entaille) sur les côtés soit une inclusion cylindrique permettant de mieux contrôler l'apparition des bandes de cisaillement dans un sable.

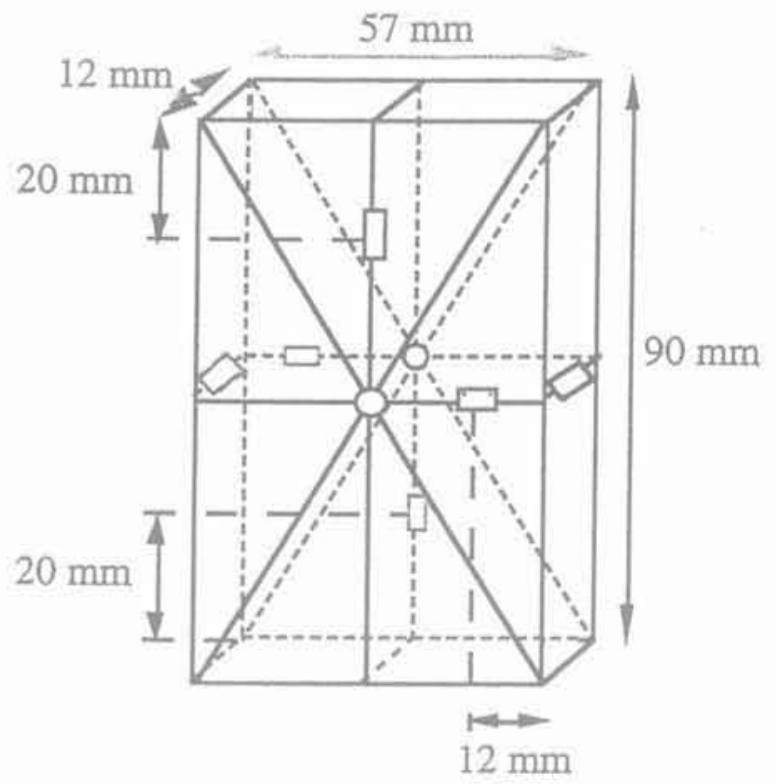

FG.5 Éprouvette prismatique percée d'un trou circulaire de diamètre $3 \mathrm{~mm}$. Geometry of prismatic samples containing hole-strain gages disposition.

\section{Comportement mécanique et seuil de localisation pour les plaques percées}

Un exemple de réponses mécaniques obtenues sur les éprouvettes percées est présenté sur la figure 6. Les résultats obtenus dans les essais sur éprouvettes non percées sont également reproduits sur ces mêmes figures. On constate que le trou n'affecte que très peu la réponse macroscopique du matériau (courbe contraintes, déformations). En effet seule une légère diminution est enregistrée pour le module élastique (inférieure à $7 \%$ ) et pour la résistance à la rupture (moins de $4 \%$ ). Des résultats similaires obtenus pour d'autres pressions de confinement ont été présentés par Haied (1995). 


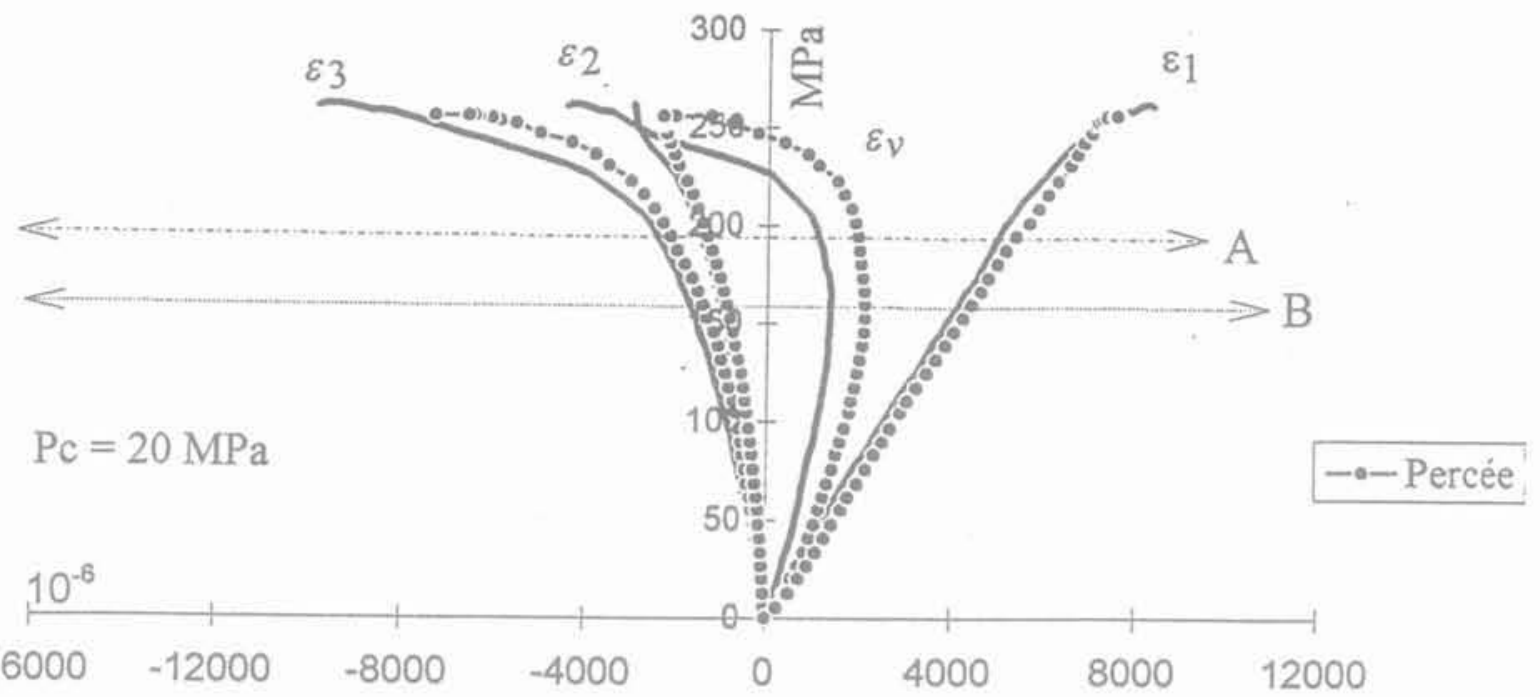

FiG.6 Exemple de résultats en compression triaxiale sur éprouvette prismatique percée. Comparaison avec les résultats sur éprouvette prismatique non percée.

Example of stress-strain curve for triaxial test on prismatic samples with hole. Comparison with results on sample without hole.
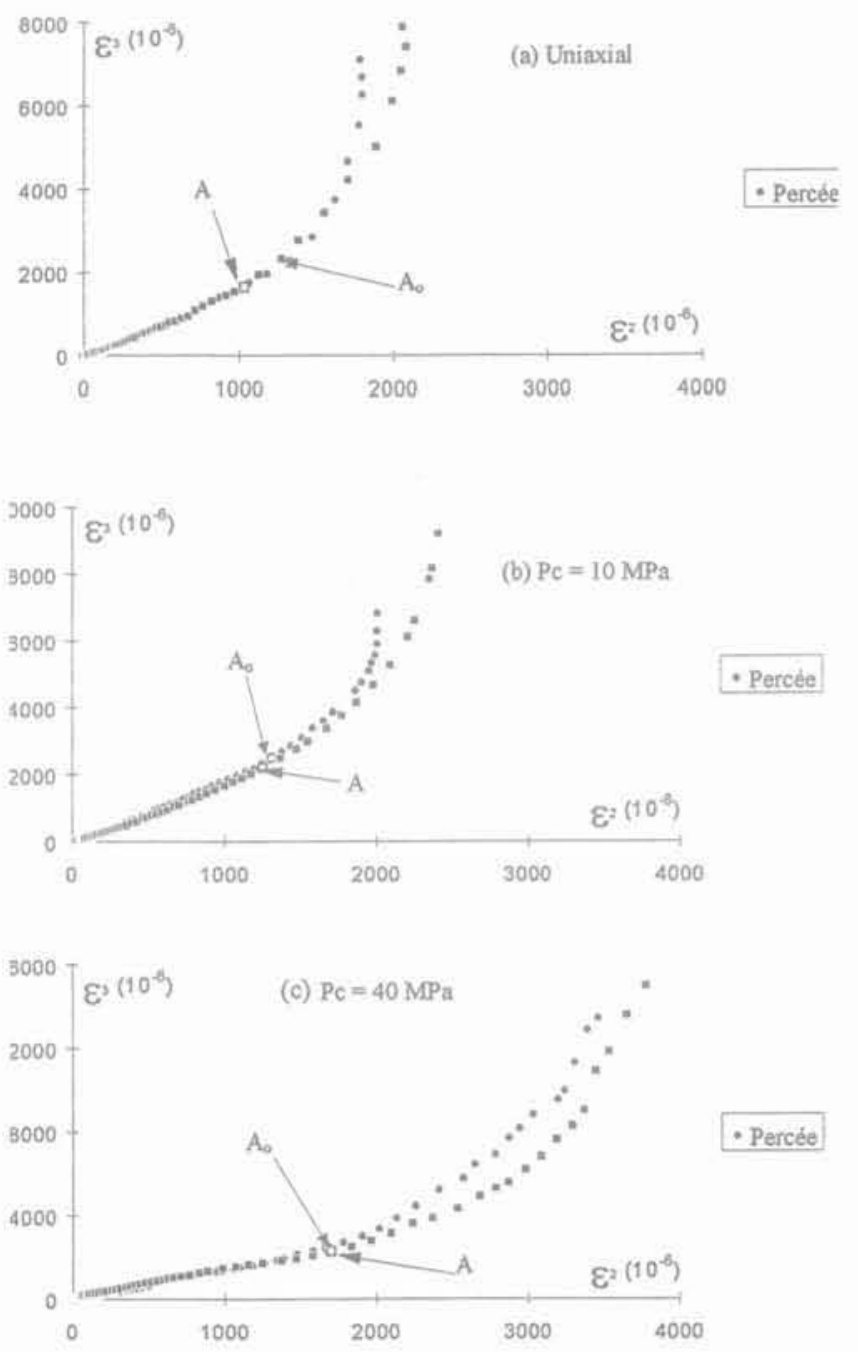

FIG. 7 Courbers $\varepsilon, \varepsilon$, dans les essais triaxiaux sur éprouvette prismatique percée. Comparaison avec les résultats des essais sur éprouvettes non avec les rées.

percées.

Curves $\varepsilon, \varepsilon$, in prismatic samples containing hole. Point $A$ indicates the onset of strain inhomogeneity. Comparisons with previous results on unpierced samples.
Des exemples de courbes $\varepsilon_{2}, \varepsilon_{3}$ pour les échantillons percés et non percés sont représentés par pression de confinement sur les figures 7 . Le seuil de localisation, obtenu pour les éprouvettes percées est noté $A_{0}$. La comparaison entre les points A (seuil dans les éprouvettes non percées) et $A_{0}$ indique la proximité de ces seuils (voir figure 7). Dans l'essai uniaxial, il correspond à $86 \%$ de la résistance à la rupture. 11 se situe respectivement à 82,76 (voir figure 6 pour ce cas), 75 et $73 \%$ de la résistance à la rupture dans les essais triaxiaux à 10 , 20, 30 et $40 \mathrm{MPa}$ de pression de confinement.

\section{2}

\section{Amorçage et caractéristiques des bandes de localisation}

L'analyse des lignes de rupture observées dans les essais sur éprouvettes percées montre le rôle important joué par l'imperfection et la pression de confinement dans l'amorçage des bandes de localisation. Toutes les éprouvettes rompues indiquent que ces bandes se sont propagées à partir de deux points diamétralement opposés de la frontière du trou circulaire. Ces deux points d'amorçage se situent aux extrémités du diamètre parallèle à l'axe de chargement dans le cas de l'essai de compression uniaxiale (Fig. 8a) et sont localisés aux extrémités du diamètre perpendiculaire à l'axe de chargement dans le cas des essais triaxiaux. La figure $8 \mathrm{~b}$ montre, par exemple, l'état de la bande à $95 \%$ du pic de contraintes dans l'essai triaxial à $20 \mathrm{MPa}$ de confinement. L'examen des lignes de rupture montre également que le trou joue un rôle important tant au niveau de l'inclinaison que de l'épaisseur moyenne des bandes. On note en particulier, une diminution appréciable de la valeur de l'angle qui oriente la bande par rapport à la direction de la contrainte principale majeure. Bien que la tendance à augmenter avec le confinement soit toujours observée, cet angle varie de 24 à $28^{\circ}$ dans les éprouvettes percées. S'agissant des largeurs de bandes, elles subissent une augmentation. Elles sont de l'ordre de 3 à $4 \mathrm{~mm}$ à faible pression de confinement ( $\mathrm{PC}=$ $10 \mathrm{MPa}$ ) et font moins d'un millimètre (comme dans le cas des éprouvettes non percées) pour $\mathrm{PC}=30$ ou $40 \mathrm{MPa}$. 


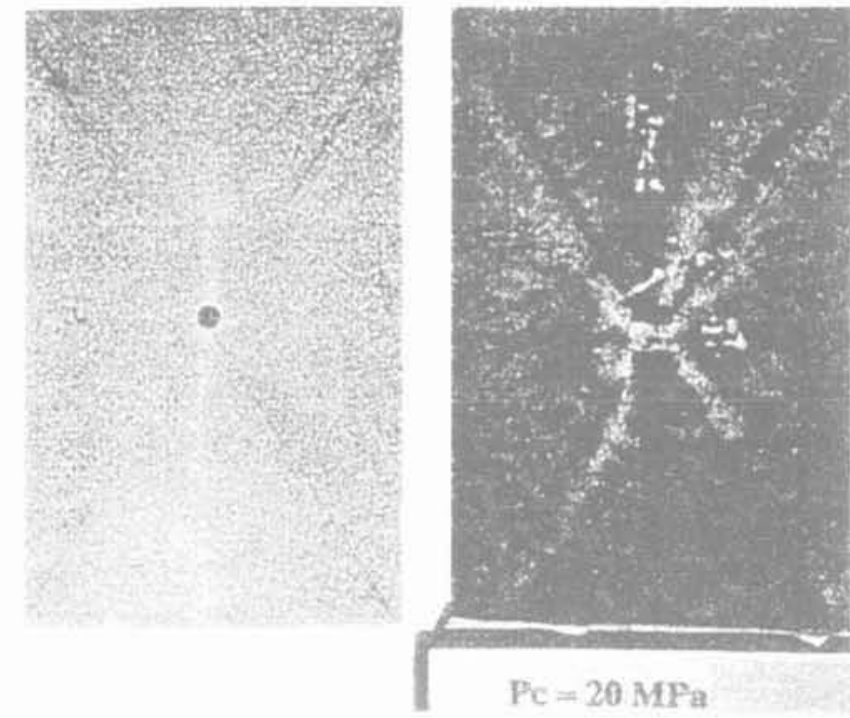

FIG. \& Exemples d'amorçage de bande de localisation dans les éprouvettes percées. a) cas uniaxial; b) cas triaxial à $\mathrm{Pc}=20 \mathrm{MPa}$. Examples of shear band in pierced samples, a) uniaxial case; b) triaxial case.

\section{5}

\section{Seuils expérimentaux de localisation: effets de la pression de confinement}

\section{- En terme de contraintes}

La figure 9 rassemble les résultats concernant l'évolution des déviateurs de contrainte au pic et au seuil de localisation A en fonction de la pression de confinement $\left(P_{f}=\sigma_{3}\right)$. On note que dans l'intervalle de pression de confinement étudié $(0,40 \mathrm{MPa})$, la résistance à la rupture et le seuil de localisation varient de manière quasi linéaire avec $P$. La pente plus faible dans la relation donnant la contrainte déviatorique au seuil A illustre qualitativement la tendance du pic a être retardé par rapport au seuil de localisation lorsque la pression de confinement augmente. Les deux relations linéaires permettent également de prévoir que, pour une pression de confinement très élevée, l'instant où la localisation se produirait tendra vers $70 \%$ (limite du rapport entre les deux approximations, pour $\sigma_{3}$ très élevé) de la résistance à la rupture.

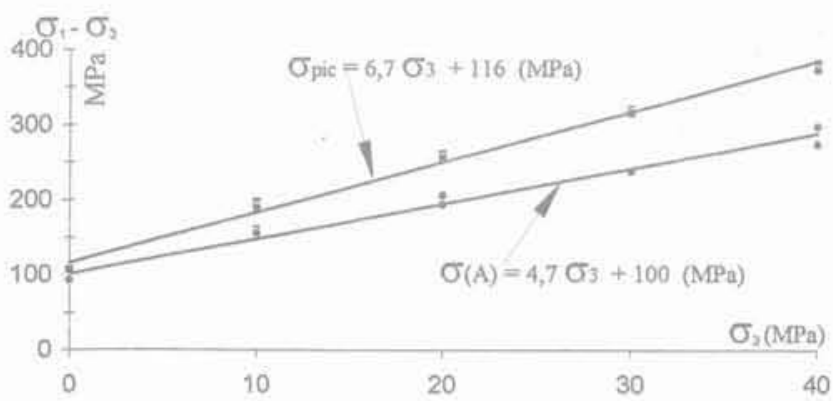

F16.9 Variation du déviateur de contraintes au seuil de localisation en fonction de la pression de confinement $\sigma_{3}=P$.

Deviatoric stress at the onset of localization as function of confining pressure.

\section{- En terme de déformations}

Comme nous l'avons déjà souligné au $\$ 3.3$, le niveau de déformation (pour chacune des trois directions) enregistré au seuil de localisation semble peu dépendant de la pression de confinement (Fig. 10). Ceci n'est pas le cas pour les déformations à la rupture macroscopique. Les valeurs moyennes $\left( \pm 5.10^{-5}\right)$ des déformations au seuil sont données dans le tableau I. Pour juger des dispersions, l'écart type et la moyenne des écarts sont fournis à titre indicatif. Le résultat obtenu (quasi-constance des déformations au seuil de localisation) indique l'intérêt de critères basés sur la déformation (d'extension) critique comme celui proposé par Stacey (1981) pour décrire la rupture fragile des roches.

TABLEAUI Valeurs moyennes et écarts des déformations au seuil de localisation. Average values of strains at localisation.

\begin{tabular}{c|c|c|c}
\hline Déformations $\left(1^{-5}\right)$ & $<e$ & Ecart-type & Écart moyen \\
Axiale $\varepsilon_{1}$ & 5,5 & 0,3 & 0,2 \\
\hline Transversale $\varepsilon_{2}$ & 1,5 & 0,2 & 0,2 \\
\hline Latérale $\varepsilon_{3}$ & 2,2 & 0,3 & 0,2 \\
\hline
\end{tabular}

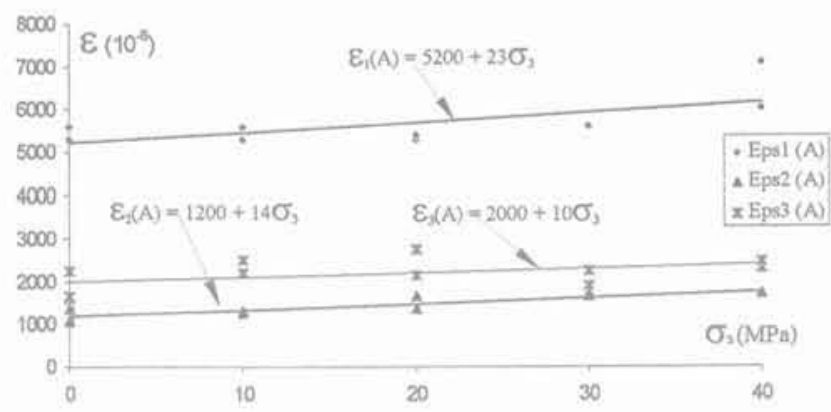

FG. 10 Variation des déformations au seuil de localisation en fonction de la pression de confinement $\sigma_{3}=P$.

Strains level at the onset of localization as function of confining pressure.

\section{Conclusion}

Outre la preuve de l'efficacité de la méthodologie expérimentale (mesures multiples de déformations) mise en œuvre, les études réalisées ont permis de caractériser les bandes de localisation des déformations dans le grès de Fontainebleau. Malgré sa simplicité cette méthodologie s'est révélé particulièrement intéressante dans les essais de compression triaxiale réalisés sur des éprouvettes prismatiques. Dans le cas des essais sur éprouvettes prismatiques non percées on note que, lorsque la pression de confinement augmente, le pic a tendance à être retardé par rapport au seuil de localisation. Lorsque le confinement augmente. on note également une légère augmentation de l'inclinaison des bandes de cisaillement par rapport à la contrainte principale majeure. Les largeurs de bande varient de à 1 à $2 \mathrm{~mm}$; elles sont difficiles à évaluer de 
manière objective, les bandes étant souvent délimitées par une zone blanchâtre à contour variable.

Afin de mieux maîtriser les conditions d'amorçage de localisation, des essais ont été ensuite réalisés sur des éprouvettes prismatiques contenant une ouverture cylindrique de faible diamètre. Il n'a pas été enregistré de modification notable dans les réponses macroscopiques du matériau et dans les seuils de localisation. En revanche les orientations moyennes des bandes ont nettement diminué $\left(25-28^{\circ}\right)$. Enfin, sur la base de l'ensemble des essais sur éprouvettes prismatiques (percées ou non), on obtient des variations linéaires des résistances et des seuils de localisation en fonction de la pression de confinement, ainsi qu'une quasi-constance des niveaux de déformations au seuil de localisation.

Les travaux expérimentaux en cours concernent deux aspects: (i) corrélations de nos constatations à I'aide d'observations au MEB. Une première partie de ce travail déjà entamé a été présentée par Haied (1995); (ii) recoupement des données quantitatives à l'aide d'une autre technique de détection de la localisation (p. ex. émission acoustique ou speckle laser).

\section{Bibliographie}

Bascoul A. Benaila E.H. Bertaud Y. Fond C., Massat M., Torrentí J.-M. Analysis of localization in concrete and rocks through stereophotogrametry, speckle laser and replica. Cement and Concrete Research, vol. $26, n^{\circ} 6$, p. 1340 1350, 1993.

Bieniawski Z.T. - Mechanism of brittle fracture of rock. Part I. Theory of the fracture process. Part Il. Experimental study. Int. J. Rock Mech. Min. Sci., vol. 4 , p. 395-430, 1967.

Desrues J. - La localisation de la déformation dans les milieux granulaires. Thèse de doctorat d'Etat, Université de Grenoble, 1984.

Hadley K. - Azimutal variation of dilatancy.
J. Geophys, Res., vol. 80, p. 4845-4850, 1975.

Haied A., Kondo D., Henry J,-P, - Experi. mental detection of shear bands in a sandstone. Assessment and prevention of failure phenomena in rock enginee. ring. p. 149-154, 1993

Haied A. - Etude expérimentale de la rupture en mode de déformations localisé d'un grès. Thèse de doctorat, Universite de Lille I, 1995.

Nadia A. - Theory of flow and fracture of solids. McGraw-Hill, p. 275-296, 1950.

Ord A., Vardoulakis I.G., Kajewski R. Shear band in Godsford sandstone. Int J. Rock. Mech. Min. Sci., vol, 28, p. 397 409. 1991.
Rudnicki J.W. Rice J.R. - Conditions of localization of deformation in pressuresensitive dilatant materials. J. Mech. Phys. Solidis, 23, p. 371-394, 1975.

Santarelli F.J. - Étude expérimentale de lá bifurcation dans les roches. Revue Fran. çaise de géotechnique, vol. 50, p. 61-70, 1990.

Stacey T.R. - A simple extension strain criterion for fracture of brittle rock. Int, J. Rock Mech. Min. Sci. \& Geomech. Abstr. vol. 18, p. $469-474,1981$.

Vardoulakis I., Graf B. - Imperfection sensitivity of the biaxial test on dry sand Proc. IUTAM Conf. Deformation and failure of granular materials, Deift, Rotterdam, Balkema, p. 485-491, 1982. 